TAIWANESE JOURNAL OF MATHEMATICS

Vol. 17, No. 4, pp. 1227-1244, August 2013

DOI: $10.11650 / \mathrm{tjm} .17 .2013 .2675$

This paper is available online at http://journal.taiwanmathsoc.org.tw

\title{
DUALITY AND FARKAS-TYPE RESULTS FOR DC INFINITE PROGRAMMING WITH INEQUALITY CONSTRAINTS
}

\author{
Xiang-Kai Sun*, Sheng-Jie Li and Dan Zhao
}

\begin{abstract}
In this paper, a DC infinite programming problem with inequality constraints is considered. By using the method of Fenchel conjugate functions, a dual scheme for the DC infinite programming problem is introduced. Then, under suitable conditions, weak and strong duality assertions are obtained. Moreover, by using the obtained duality assertions, some Farkas-type results which characterize the optimal value of the DC infinite programming problem are given. As applications, the proposed approach is applied to conic programming problems.
\end{abstract}

\section{INTRODUCTION}

In this paper, we deal with a new class of DC infinite programming with inequality constraints given in the following form:

$$
(P)\left\{\begin{array}{c}
\min f(x)-g(x) \\
\text { s.t. } h_{t}(x) \leq 0, t \in T, \text { and } x \in C,
\end{array}\right.
$$

where $T$ is a nonempty (possibly infinite) index set, $C$ is a nonempty convex subset of a locally convex space $X$, and $f, g, h_{t}: X \rightarrow \bar{R}:=R \cup\{+\infty\}, t \in T$, are proper lower semicontinuous, and convex functions. Throughout this paper, we always assume that

$$
\mathcal{A}:=C \cap\left\{x \in X: h_{t}(x) \leq 0 \text {, for all } t \in T\right\} \neq \emptyset .
$$

Moreover, we refer to such problem as DC infinite programming, since the index set $T$ is infinite, and the objective function is the difference of two convex functions.

Received November 17, 2012, accepted January 14, 2013.

Communicated by Jen-Chih Yao.

2010 Mathematics Subject Classification: 49N15, 90C32, 90C46.

Key words and phrases: Duality, Conjugate function, Farkas-type results, DC infinite programming.

This research was partially supported by the National Natural Science Foundation of China (Grant numbers: 11171362 and 11201509).

*Corresponding author. 
When the space $X$ is finite dimensional, optimization problems with infinite inequality constraints is called semi-infinite programming.

If $g(x)=0$, then, the problem $(P)$ reduces to the following convex programming involving infinite constraints:

$$
\left(P_{0}\right)\left\{\begin{array}{l}
\min f(x) \\
\text { s.t. } h_{t}(x) \leq 0, t \in T, \text { and } x \in C .
\end{array}\right.
$$

This problem $\left(P_{0}\right)$ has numerous applications in Chebyshev approximation, robotics, mathematical physics, engineering design, optimal control, cooperative games, transportation problems and robust optimization. It has been studied extensively under various conditions imposed on the objective function, or the constraint conditions, see [1-11] and the references cited therein.

DC programming problems are important from both viewpoints of optimization theory and applications. Many important results have been extensively obtained in the last decades, see [12-20] and the references therein. On the one hand, such problems being heavily non-convex can be considered as a special class of quasi-differentiable programming [21] and thus advanced techniques of variational analysis can be applied, see $[21,22]$. On the other hand, the special convex structure of both plus function and minus function offers the possibility to use powerful tools of convex analysis in the study of DC programming problems.

Now, there has been an increasing interest in research in dual problems. The reason is that they may be easier to solve than the primal ones (which is sometimes really the case) or that at least they furnish additional information about the primal ones. At the same time, various dual schemes are proposed for optimization problems, variational inequalities, and so on. Here, we specially mention the works on duality defined via convexification techniques due to Boţ et al. [13], Dinh et al. [15, 16] and Fang et al. [20]. By using the interiority condition, Boţ et al. [13] investigated Fenchel-Lagrange duality results and Farkas-type lemmas for DC programming with DC objective functions and finitely many DC inequality constraints. Dinh et al. $[15,16]$ considered the some types Fenchel-Lagrange dual problems for DC conical problems in terms of the epigraph closure condition. Fang et al. [20] investigated Lagrange duality results and extended Farkas lemmas for DC infinite programming. However, there is no paper to deal with Fenchel-Lagrange dual problems for DC programming problems with DC objective functions and infinitely many inequality constraints. Then, the purpose of this paper is to obtain strong duality characterizations for $(P)$ and then to investigated the Farkas results of $(P)$, including cone programming problems [15, $16,25-28]$, which have received a great deal of attention in recent years. In this paper, we first recall the closedness qualification condition $(C Q C)$ introduced in [24]. We also give some characterizations of this condition. Note that constraint qualifications involving subdifferentials have been studied and extensively used, see [25, 26, 29-33] and the references therein. Our main aims in this paper is to use these constraint 
qualifications to provide complete characterizations for the duality results and Farkastype results for problem $(P)$.

The paper is organized as follows. In Section 2, we recall some notions and give some preliminary results. In Section 3, we construct the dual problems to $(P)$, and prove weak duality and strong duality assertions. By using the duality assertions, we obtain some Farkas-type results for the problem $(P)$. In Section 4, we apply these problems to conic programming problems.

\section{Mathematical Preliminaries}

Throughout this paper, let $X$ be a real locally convex vector spaces with its continuous dual spaces $X^{*}$, endowed with the weak* topologies $w\left(X^{*}, X\right)$. We always use the notation $\langle\cdot, \cdot\rangle$ for the canonical paring between $X$ and $X^{*}$. Let $D$ be a set in $X$, the interior (resp. closure, convex hull, convex cone hull) of $D$ is denoted by int $D$ (resp. cl $D$, co $D$, cone $D$ ). Thus if $W \subseteq X^{*}$, then cl $W$ denotes the weak* closure of $W$. We shall adopt the convention that cone $D=\{0\}$ when $D$ is an empty set. Let $D^{*}=\left\{x^{*} \in X^{*}:\left\langle x^{*}, x\right\rangle \geq 0, \forall x \in X\right\}$ be the dual cone of $D$. The indicator function $\delta_{D}: X \rightarrow \bar{R}$ of $X$ is defined by

$$
\delta_{D}(x)= \begin{cases}0, & \text { if } x \in D \\ +\infty, & \text { if } x \notin D .\end{cases}
$$

The support function $\sigma_{D}: X^{*} \rightarrow \bar{R}$ of $D$ is defined by

$$
\sigma_{D}\left(x^{*}\right)=\sup _{x \in D}\left\langle x^{*}, x\right\rangle .
$$

Further, let $R^{T}$ be the product space of $\lambda=\left(\lambda_{t}\right)_{t \in T}$ with $\lambda_{t} \in R$ for all $t \in T$, let $R^{(T)}$ be collection of $\lambda \in R^{T}$ with $\lambda_{t} \neq 0$ for finitely many $t \in T$, and let $R_{+}^{(T)}$ be the positive cone in $R^{(T)}$ defined by

$$
R_{+}^{(T)}:=\left\{\lambda \in R^{(T)}: \lambda_{t} \geq 0 \text { for all } t \in T\right\} .
$$

Given $u \in R^{T}$ and $\lambda \in R^{(T)}$, and denoting supp $\lambda:=\left\{t \in T: \lambda_{t} \neq 0\right\}$, we have

$$
\langle\lambda, u\rangle:=\sum_{t \in T} \lambda_{t} u_{t}=\sum_{t \in \operatorname{supp} \lambda} \lambda_{t} u_{t} .
$$

Let $l: X \rightarrow \bar{R}$ be a proper convex function. The effective domain and the epigraph are defined by

$$
\operatorname{dom} l=\{x \in X: l(x)<+\infty\}
$$

and

$$
\text { epi } l=\{(x, r) \in X \times R: l(x) \leq r\} \text {, }
$$


respectively. $l$ is said to be proper if its effective domain is nonempty and $l(x)>-\infty$, for all $x \in X$. The conjugate function $l^{*}: X^{*} \rightarrow \bar{R}$ of $l$ is defined by

$$
l^{*}\left(x^{*}\right)=\sup _{x \in X}\left\{\left\langle x^{*}, x\right\rangle-l(x)\right\} .
$$

Let $\bar{x} \in \operatorname{dom} l$. For any $\varepsilon \geq 0$, the $\varepsilon$-subdifferential of $l$ at $\bar{x}$ is the convex set defined by

$$
\partial_{\varepsilon} l(\bar{x})=\left\{x^{*} \in X^{*}: l(x) \geq l(\bar{x})+\left\langle x^{*}, x-\bar{x}\right\rangle-\varepsilon, \forall x \in X\right\} .
$$

When $\bar{x} \notin \operatorname{dom} l$, we define that $\partial_{\varepsilon} l(\bar{x})=\emptyset$. If $\varepsilon=0$, the set $\partial l(\bar{x}):=\partial_{0} l(\bar{x})$ is the classical subdifferential of convex analysis, that is,

$$
\partial l(\bar{x})=\left\{x^{*} \in X^{*}: l(x) \geq l(\bar{x})+\left\langle x^{*}, x-\bar{x}\right\rangle, \forall x \in X\right\} .
$$

It is easy to prove that for any $\bar{x} \in \operatorname{dom} l$ and $x^{*} \in X^{*}$, the following inequality holds

$$
l(\bar{x})+l^{*}\left(x^{*}\right) \leq\left\langle x^{*}, \bar{x}\right\rangle+\varepsilon \Leftrightarrow x^{*} \in \partial_{\varepsilon} l(\bar{x}) .
$$

Moreover, following [34], we have

$$
\text { epi } l^{*}=\bigcup_{\varepsilon \geq 0}\left\{\left(x^{*},\left\langle x^{*}, \bar{x}\right\rangle+\varepsilon-l(\bar{x})\right): x^{*} \in \partial_{\varepsilon} l(\bar{x})\right\} \text {. }
$$

Let $E$ be a convex set of $X$. the $\varepsilon$-normal cone to $E$ at a point $\bar{x} \in E$ is defined by

$$
N_{\varepsilon}(E, \bar{x})=\left\{x^{*} \in X^{*}:\left\langle x^{*}, x-\bar{x}\right\rangle \leq \varepsilon, \text { for any } x \in E\right\} .
$$

If $\varepsilon=0, N_{0}(E, \bar{x})$ is the normal cone $N(E, \bar{x})$ of convex analysis. Moreover, it is easy to see that $N_{\varepsilon}(E, \bar{x})=\partial_{\varepsilon} \delta_{E}(\bar{x})$.

Definition 2.1. Let the functions $l_{1}, l_{2}: X \rightarrow \bar{R}$ be given. The infimal convolution function of $l_{1}, l_{2}$ is the function $l_{1} \square l_{2}: X \rightarrow \bar{R}$ defined by

$$
\left(l_{1} \square l_{2}\right)(x)=\inf _{x=x_{1}+x_{2}}\left\{l_{1}\left(x_{1}\right)+l_{2}\left(x_{2}\right)\right\} .
$$

We say that $l_{1} \square l_{2}$ is exact at $x \in X$ if there exist $x_{1}, x_{2} \in X$ such that $\left(l_{1} \square l_{2}\right)(x)=$ $l_{1}\left(x_{1}\right)+l_{2}\left(x_{2}\right)$. Furthermore, we call $l_{1} \square l_{2}$ exact if it is exact at every $x \in X$.

Now, let us recall the following result which will be used in the following section.

Lemma 2.1. (See [30]). Let $l_{1}, l_{2}: X \rightarrow \bar{R}$ be proper, convex and lower semicontinuous functions such that $\operatorname{dom} l_{1} \cap \operatorname{dom} l_{2} \neq \emptyset$. Then, the following relation holds

$$
\text { epi }\left(l_{1}+l_{2}\right)^{*}=\operatorname{cl}\left(\operatorname{epi}\left(l_{1}^{*} \square l_{2}^{*}\right)\right)=\operatorname{cl}\left(\operatorname{epi} l_{1}^{*}+\operatorname{epi} l_{2}^{*}\right),
$$

where the closure is taken in the product topology of $\left(X^{*}, \tau\right) \times R$, for any locally convex topology $\tau$ on $X^{*}$ giving $X$ as dual. 
Lemma 2.2. (See [35]). Let $l_{1}, l_{2}: X \rightarrow \bar{R}$ be two proper, convex and lower semicontinuous functions. Then

$$
\inf _{x \in X}\left\{l_{1}(x)-l_{2}(x)\right\}=\inf _{x^{*} \in X^{*}}\left\{l_{2}^{*}\left(x^{*}\right)-l_{1}^{*}\left(x^{*}\right)\right\} .
$$

\section{MaIn Results}

In this section, we first construct the dual problems of $(P)$, and present the duality assertions. Then, by using the duality assertions, we investigated some Farkas-type results for the problem $(P)$. Now, we first introduce the following closedness condition which completely characterize the duality of the problem $(P)$.

Definition 3.1. (See [24]). The problem $(P)$ is said to satisfy the closedness qualification condition $(C Q C)$, if the set

$$
\text { (CQC) epi } f^{*}+\text { cone }\left(\bigcup_{t \in T} \text { epi } h_{t}^{*}\right)+\text { epi } \delta_{C}^{*}
$$

is weak* closed in the space $X^{*} \times R$.

Remark 3.1. Let $\mathcal{A}:=C \cap\left\{x \in X: h_{t}(x) \leq 0\right.$, for all $\left.t \in T\right\}$. Then, $\mathcal{A}$ is a convex subset of $X$ and the following relation established in [8] holds:

$$
\operatorname{epi} \delta_{\mathcal{A}}^{*}=\operatorname{cl}\left(\operatorname{cone}\left(\bigcup_{t \in T} \text { epi } h_{t}^{*}\right)+\operatorname{epi} \delta_{C}^{*}\right)
$$

The next lemma provides several characterizations of the $(C Q C)$ property for $\mathrm{DC}$ infinite programs. The equivalence results of this lemma extend to the general DC infinite program setting $(P)$ those recently obtained in [16, Theorem 3.1] for coneconstrained programs. Moreover, this condition will be crucial in the sequel and it also deserves some attention for its independent interest.

Lemma 3.1. (See [24]). The following statements are equivalent:

(i) Condition $(C Q C)$ holds.

(ii) For any $x^{*} \in X^{*}$,

$$
\left(f+\delta_{\mathcal{A}}\right)^{*}\left(x^{*}\right)
$$

(3)

$$
=\min _{\lambda \in R_{+}^{(T)}} \min _{\substack{u^{*}, v_{t}^{*} \in X^{*} \\ t \in \operatorname{supp} \lambda}}\left\{f^{*}\left(u^{*}\right)+\sum_{t \in \operatorname{supp} \lambda} \lambda_{t} h_{t}^{*}\left(v_{t}^{*}\right)+\delta_{C}^{*}\left(x^{*}-u^{*}-\sum_{t \in \operatorname{supp} \lambda} \lambda_{t} v_{t}^{*}\right)\right\} .
$$

(iii) For any $\bar{x} \in \mathcal{A} \cap \operatorname{dom} f$ and $\varepsilon \geq 0$, we have the equality 


$$
\begin{aligned}
& \partial_{\varepsilon}\left(f+\delta_{\mathcal{A}}\right)(\bar{x})=\bigcup_{\lambda \in R_{+}^{(T)}} \bigcup_{\substack{\varepsilon_{1}+\sum_{1}, \varepsilon_{t} \geq 0, t \in \operatorname{supp} \lambda \\
\varepsilon_{1} \operatorname{supp} \lambda \lambda_{t}\left[\varepsilon_{t}-h_{t}(\bar{x})\right]+\varepsilon_{2}=\varepsilon}} \\
& \left\{\partial_{\varepsilon_{1}} f(\bar{x})+\sum_{t \in \operatorname{supp} \lambda} \lambda_{t} \partial_{\varepsilon_{t}} h_{t}(\bar{x})+N_{\varepsilon_{2}}(C, \bar{x})\right\} .
\end{aligned}
$$

Now, we will establish a dual problem to $(P)$ and give the sufficient conditions which ensure that the optimal objective value of the primal problem coincides with the optimal objective value of the dual problem. In order to introduce the dual scheme for problem $(P)$, we need the following lemma.

Lemma 3.2. (See $[7,18]$ ). For any $x$ feasible to the problem $(P)$, we have

$$
g(x)=\sup _{x^{*} \in X^{*}}\left\{\left\langle x^{*}, x\right\rangle-g^{*}\left(x^{*}\right)\right\} .
$$

Proof. Since $g$ is proper convex, and lower semicontinuous function, we have

$$
g(x)=g^{* *}(x)=\sup _{x^{*} \in X^{*}}\left\{\left\langle x^{*}, x\right\rangle-g^{*}\left(x^{*}\right)\right\} .
$$

This completes the proof.

Note that the problem $(P)$ can be rewritten as:

$$
(P) \inf _{x \in X}\left\{f(x)-g(x)+\delta_{\mathcal{A}}(x)\right\} .
$$

Since $g$ is lower semicontinuous, the standard convexification technique can be applied. Then, by Lemma 3.2, the problem $(P)$ becomes:

$$
\text { (P) } \quad \inf _{x^{*} \in X^{*}} \inf _{x \in X}\left\{f(x)+\delta_{\mathcal{A}}(x)+g\left(x^{*}\right)-\left\langle x^{*}, x\right\rangle\right\} .
$$

Note that for any $x^{*} \in X^{*}$, the inner infimum of the last formula

$$
\left(P^{x^{*}}\right) \quad \inf _{x \in X}\left\{f(x)+\delta_{\mathcal{A}}(x)+g\left(x^{*}\right)-\left\langle x^{*}, x\right\rangle\right\}
$$

is a convex optimization problem, and its Fenchel-Lagrange dual problem is

$$
\left(D^{\left.x^{*}\right)} \sup _{\substack{\lambda \in R_{+}^{(T)}, u^{*}, v_{t}^{*} \in X^{*}, t \in \operatorname{supp} \lambda}}\left\{g^{*}\left(x^{*}\right)-f^{*}\left(u^{*}\right)-\sum_{t \in \operatorname{supp} \lambda} \lambda_{t} h_{t}^{*}\left(v_{t}^{*}\right)-\delta_{C}^{*}\left(x^{*}-u^{*}-\sum_{t \in \operatorname{supp} \lambda} \lambda_{t} v_{t}^{*}\right)\right\} .\right.
$$

Thus, this reformulation motivates us to define the following dual problem of $(P)$ :

(D) $\inf _{x^{*} \in X^{*}} \sup _{\substack{\lambda \in R_{+}^{(T)}, u^{*}, v_{t}^{*} \in X^{*}, t \in \operatorname{supp} \lambda}}\left\{g^{*}\left(x^{*}\right)-f^{*}\left(u^{*}\right)-\sum_{t \in \operatorname{supp} \lambda} \lambda_{t} h_{t}^{*}\left(v_{t}^{*}\right)-\delta_{C}^{*}\left(x^{*}-u^{*}-\sum_{t \in \operatorname{supp} \lambda} \lambda_{t} v_{t}^{*}\right)\right\}$. 
Here and throughout this paper, following Fang et al. [17, 19] and Zalinescu [36], we adapt the convention that

$$
\begin{gathered}
(+\infty)-(+\infty)=(-\infty)-(-\infty)=(+\infty)+(-\infty)=(-\infty)+(+\infty)=+\infty \\
0 \cdot(+\infty)=+\infty \text { and } 0 \cdot(-\infty)=0
\end{gathered}
$$

Now, we will study the weak dualities and the strong dualities between $(P)$ and $(D)$. For the optimization problem $(P)$, we denote by $\operatorname{val}(P)$ its optimal objective value and this notation is extended to the optimization problems that we use in this paper.

Definition 3.2. We say that

(i) the weak duality between $(P)$ and $(D)$ holds, if $\operatorname{val}(P) \geq \operatorname{val}(D)$.

(ii) the strong duality between $(P)$ and $(D)$ holds, if $\operatorname{val}(P)=\operatorname{val}(D)$, and for any $x^{*} \in X^{*}$ satisfying $\operatorname{val}\left(D^{x^{*}}\right)=\operatorname{val}(D)$, the dual problem $\left(D^{x^{*}}\right)$ has an optimal solution.

Remark 3.2. It is easy to see that the strong duality between $(P)$ and $(D)$ holds if and only if

$$
\begin{aligned}
\operatorname{val}(P)= & \inf _{x^{*} \in X^{*}} \max _{\substack{\lambda \in R_{+}^{(T)}, u^{*}, v_{t}^{*} \in X^{*}, t \in \operatorname{supp} \lambda}} \\
& \left\{g^{*}\left(x^{*}\right)-f^{*}\left(u^{*}\right)-\sum_{t \in \operatorname{supp} \lambda} \lambda_{t} h_{t}^{*}\left(v_{t}^{*}\right)-\delta_{C}^{*}\left(x^{*}-u^{*}-\sum_{t \in \operatorname{supp} \lambda} \lambda_{t} v_{t}^{*}\right)\right\} .
\end{aligned}
$$

Theorem 3.1. The weak duality between $(P)$ and $(D)$ is fulfilled, namely, $\operatorname{val}(P) \geq$ $\operatorname{val}(D)$.

Proof. It follows from the definition of conjugate functions that for any $\lambda \in R_{+}^{T}$, $t \in \operatorname{supp} \lambda, x^{*}, u^{*}, v_{t}^{*} \in X^{*}$, and $x \in \mathcal{A}$, we have

$$
\begin{aligned}
& g^{*}\left(x^{*}\right)-f^{*}\left(u^{*}\right)-\sum_{t \in \operatorname{supp} \lambda} \lambda_{t} h_{t}^{*}\left(v_{t}^{*}\right)-\delta_{C}^{*}\left(x^{*}-u^{*}-\sum_{t \in \operatorname{supp} \lambda} \lambda_{t} v_{t}^{*}\right) \\
\leq & g^{*}\left(x^{*}\right)-\left\langle u^{*}, x\right\rangle+f(x)-\sum_{t \in \operatorname{supp} \lambda} \lambda_{t}\left\langle v_{t}^{*}, x\right\rangle \\
& +\sum_{t \in \operatorname{supp} \lambda} \lambda_{t} h_{t}(x)-\left\langle x^{*}-u^{*}-\sum_{t \in \operatorname{supp} \lambda} \lambda_{t} v_{t}^{*}, x\right\rangle \\
\leq & g^{*}\left(x^{*}\right)-\left\langle x^{*}, x\right\rangle+f(x)+\sum_{t \in \operatorname{supp} \lambda} \lambda_{t} h_{t}(x) .
\end{aligned}
$$


Thus, for any $x \in \mathcal{A}$, we have

$$
\begin{aligned}
& \inf _{x^{*} \in X^{*}} \sup _{\substack{\lambda \in R_{+}^{(T)}, u^{*}, v_{t}^{*} \in X^{*}, t \in \operatorname{supp} \lambda}}\left\{g^{*}\left(x^{*}\right)-f^{*}\left(u^{*}\right)\right. \\
& \left.-\sum_{t \in \operatorname{supp} \lambda} \lambda_{t} h_{t}^{*}\left(v_{t}^{*}\right)-\delta_{C}^{*}\left(x^{*}-u^{*}-\sum_{t \in \operatorname{supp} \lambda} \lambda_{t} v_{t}^{*}\right)\right\} \\
\leq & \inf _{x^{*} \in X^{*}}\left\{g^{*}\left(x^{*}\right)-\left\langle x^{*}, x\right\rangle\right\}+f(x)+\sum_{t \in \operatorname{supp} \lambda} \lambda_{t} h_{t}(x) \\
= & -g^{* *}(x)+f(x)+\sum_{t \in \operatorname{supp} \lambda} \lambda_{t} h_{t}(x) \\
\leq & f(x)-g(x),
\end{aligned}
$$

which means that $\operatorname{val}(P) \geq \operatorname{val}(D)$, and the proof is complete.

Theorem 3.2. If $(C Q C)$ is fulfilled, then, the strong duality between $(P)$ and $(D)$ holds, namely, $\operatorname{val}(P)=\operatorname{val}(D)$, and for any $x^{*} \in X^{*} \operatorname{satisfying} \operatorname{val}\left(D^{x^{*}}\right)=\operatorname{val}(D)$, the dual problem $\left(D^{x^{*}}\right)$ has an optimal solution.

Proof. Since the problem $(P)$ can be rewritten as

$$
\inf _{x \in X}\left\{\left(f+\delta_{\mathcal{A}}\right)(x)-g(x)\right\}
$$

it follows from Lemma 2.2 that

$$
\inf _{x \in X}\left\{\left(f+\delta_{\mathcal{A}}\right)(x)-g(x)\right\}=\inf _{x^{*} \in X^{*}}\left\{g^{*}\left(x^{*}\right)-\left(f+\delta_{\mathcal{A}}\right)^{*}\left(x^{*}\right)\right\}
$$

By Lemma 3.1 and the condition $(C Q C)$, we get

$$
\begin{aligned}
& \left(f+\delta_{\mathcal{A}}\right)^{*}\left(x^{*}\right) \\
= & \min _{\lambda \in R_{+}^{(T)}} \min _{\substack{u^{*}, v_{t}^{*} \in X^{*}, t \in \operatorname{supp} \lambda}}\left\{f^{*}\left(u^{*}\right)+\sum_{t \in \operatorname{supp} \lambda} \lambda_{t} h_{t}^{*}\left(v_{t}^{*}\right)+\delta_{C}^{*}\left(x^{*}-u^{*}-\sum_{t \in \operatorname{supp} \lambda} \lambda_{t} v_{t}^{*}\right)\right\} .
\end{aligned}
$$


This equality and (5) leads to

$$
\begin{aligned}
& \operatorname{val}(P)=\inf _{x \in X}\left\{\left(f+\delta_{\mathcal{A}}\right)(x)-g(x)\right\} \\
& =\inf _{w^{*} \in X^{*}}\left\{g^{*}\left(x^{*}\right)-\min _{\substack{\lambda \in R_{+}^{(T)}, u^{*}, v_{t}^{*} \in X^{*} \\
t \in \operatorname{supp} \lambda}}\right. \\
& \left.\left\{f^{*}\left(u^{*}\right)+\sum_{t \in \operatorname{supp} \lambda} \lambda_{t} h_{t}^{*}\left(v_{t}^{*}\right)+\delta_{C}^{*}\left(x^{*}-u^{*}-\sum_{t \in \operatorname{supp} \lambda} \lambda_{t} v_{t}^{*}\right)\right\}\right\} \\
& =\inf _{x^{*} \in X^{*}} \max _{\substack{\lambda \in R_{+}^{(T)}, u^{*}, v_{t}^{*} \in X^{*}, t \in \text { supp } \lambda}}\left\{g^{*}\left(x^{*}\right)-f^{*}\left(u^{*}\right)\right. \\
& \left.-\sum_{t \in \operatorname{supp} \lambda} \lambda_{t} h_{t}^{*}\left(v_{t}^{*}\right)-\delta_{C}^{*}\left(x^{*}-u^{*}-\sum_{t \in \operatorname{supp} \lambda} \lambda_{t} v_{t}^{*}\right)\right\} .
\end{aligned}
$$

Thus, by Remark 3.2, we have that the strong duality between $(P)$ and $(D)$ holds, and the proof is completed.

By using the duality assertions presented in the previous theorems, we can obtain the following Farkas-type results.

Theorem 3.3. If the condition $(C Q C)$ is satisfied, then, for any $\alpha \in R$, the following statements are equivalent:

(i) $x \in C, h_{t}(x) \leq 0, t \in T \Longrightarrow f(x)-g(x) \geq \alpha$.

(ii) $(0,-\alpha)+$ epi $g^{*} \subseteq$ epi $f^{*}+$ cone $\left(\bigcup_{t \in T}\right.$ epi $\left.h_{t}^{*}\right)+$ epi $\delta_{C}^{*}$.

(iii) For any $x^{*} \in X^{*}$, there exist $\lambda \in R_{+}^{(T)}, u^{*}, v_{t}^{*} \in X^{*}$ and $t \in \operatorname{supp} \lambda$ such that

$$
g^{*}\left(x^{*}\right)-f^{*}\left(u^{*}\right)-\sum_{t \in \operatorname{supp} \lambda} \lambda_{t} h_{t}^{*}\left(v_{t}^{*}\right)-\delta_{C}^{*}\left(x^{*}-u^{*}-\sum_{t \in \operatorname{supp} \lambda} \lambda_{t} v_{t}^{*}\right) \geq \alpha
$$

Proof. (i) $\Rightarrow$ (ii). Suppose that (i) holds. Then, for any $x \in \mathcal{A}, f(x) \geq g(x)+\alpha$. It follows that $\left(f+\delta_{\mathcal{A}}\right)(x) \geq g(x)+\alpha$, which means that $(g+\alpha)^{*} \geq\left(f+\delta_{\mathcal{A}}\right)^{*}$. In turn, this gives that

$$
(0,-\alpha)+\text { epi } g^{*}=\operatorname{epi}(g+\alpha)^{*} \subseteq \operatorname{epi}\left(f+\delta_{\mathcal{A}}\right)^{*}
$$


Moreover, since the condition $(C Q C)$ is satisfied, it follows from Lemma 2.1 and (2) that

$$
\begin{aligned}
& \operatorname{epi}\left(f+\delta_{\mathcal{A}}\right)^{*}=\mathrm{cl}\left(\operatorname{epi} f^{*}+\operatorname{epi} \delta_{\mathcal{A}}^{*}\right) \\
= & \operatorname{cl}\left(\operatorname{epi} f^{*}+\operatorname{cl}\left(\operatorname{cone}\left(\bigcup_{t \in T} \text { epi } h_{t}^{*}\right)+\text { epi } \delta_{C}^{*}\right)\right) \\
= & \operatorname{cl}\left(\operatorname{epi} f^{*}+\operatorname{cone}\left(\bigcup_{t \in T} \operatorname{epi} h_{t}^{*}\right)+\operatorname{epi} \delta_{C}^{*}\right) \\
= & \operatorname{epi} f^{*}+\operatorname{cone}\left(\bigcup_{t \in T} \operatorname{epi} h_{t}^{*}\right)+\operatorname{epi} \delta_{C}^{*} .
\end{aligned}
$$

Thus,

$$
(0,-\alpha)+\text { epi } g^{*} \subseteq \text { epi } f^{*}+\text { cone }\left(\bigcup_{t \in T} \text { epi } h_{t}^{*}\right)+\text { epi } \delta_{C}^{*},
$$

and (ii) holds.

(ii) $\Rightarrow$ (iii). Suppose that (ii) holds. As $\left(x^{*}, g^{*}\left(x^{*}\right)\right) \in$ epi $g^{*}$, by (ii), we have

$$
\left(x^{*}, g^{*}\left(x^{*}\right)-\alpha\right) \in \operatorname{epi} f^{*}+\text { cone }\left(\bigcup_{t \in T} \text { epi } h_{t}^{*}\right)+\text { epi } \delta_{C}^{*},
$$

Then, there exist $\lambda \in R_{+}^{(T)},\left(u^{*}, \alpha_{1}\right) \in$ epi $f^{*},\left(w^{*}, \alpha_{2}\right) \in$ epi $\delta_{C}^{*}$ and $\left(v_{t}^{*}, \beta_{t}\right) \in$ epi $h_{t}^{*}$ with $t \in \operatorname{supp} \lambda$ such that

$$
\left(x^{*}, g^{*}\left(x^{*}\right)-\alpha\right)=\left(u^{*}, \alpha_{1}\right)+\sum_{t \in \operatorname{supp} \lambda} \lambda_{t}\left(v_{t}^{*}, \beta_{t}\right)+\left(w^{*}, \alpha_{2}\right),
$$

which means that

$$
x^{*}=u^{*}+\sum_{t \in \operatorname{supp} \lambda} \lambda_{t} v_{t}^{*}+w^{*}
$$

and

$$
g^{*}\left(x^{*}\right)-\alpha=\alpha_{1}+\sum_{t \in \operatorname{supp} \lambda} \lambda_{t} \beta_{t}+\alpha_{2} .
$$

Since $f^{*}\left(u^{*}\right) \leq \alpha_{1}, h_{t}^{*}\left(v_{t}^{*}\right) \leq \beta_{t}$ and $\delta_{C}^{*}\left(w^{*}\right) \leq \alpha_{2}$, it follows from (6) and (7) that

$$
\begin{aligned}
g^{*}\left(x^{*}\right)-\alpha & \geq f^{*}\left(u^{*}\right)+\sum_{t \in \operatorname{supp} \lambda} \lambda_{t} h_{t}^{*}\left(v_{t}^{*}\right)+\delta_{C}^{*}\left(w^{*}\right) \\
& =f^{*}\left(u^{*}\right)+\sum_{t \in \operatorname{supp} \lambda} \lambda_{t} h_{t}^{*}\left(v_{t}^{*}\right)+\delta_{C}^{*}\left(x^{*}-u^{*}-\sum_{t \in \operatorname{supp} \lambda} \lambda_{t} v_{t}^{*}\right) .
\end{aligned}
$$


Thus,

$$
g^{*}\left(x^{*}\right)-f^{*}\left(u^{*}\right)-\sum_{t \in \operatorname{supp} \lambda} \lambda_{t} h_{t}^{*}\left(v_{t}^{*}\right)-\delta_{C}^{*}\left(x^{*}-u^{*}-\sum_{t \in \operatorname{supp} \lambda} \lambda_{t} v_{t}^{*}\right) \geq \alpha,
$$

and (iii) holds.

(iii) $\Rightarrow$ (i). Suppose that (iii) holds. Then, for any $x^{*} \in X^{*}$, there exist $\lambda \in$ $R_{+}^{(T)}, u^{*}, v_{t}^{*} \in X^{*}$ and $t \in \operatorname{supp} \lambda$ such that

$$
g^{*}\left(x^{*}\right)-f^{*}\left(u^{*}\right)-\sum_{t \in \operatorname{supp} \lambda} \lambda_{t} h_{t}^{*}\left(v_{t}^{*}\right)-\delta_{C}^{*}\left(x^{*}-u^{*}-\sum_{t \in \operatorname{supp} \lambda} \lambda_{t} v_{t}^{*}\right) \geq \alpha,
$$

which implies that

$$
\begin{aligned}
& \sup _{\substack{\lambda \in R_{+}^{(T)}, u^{*}, v_{t}^{*} \in X^{*}, t \in \operatorname{supp} \lambda}}\left\{g^{*}\left(x^{*}\right)-f^{*}\left(u^{*}\right)\right. \\
& \left.-\sum_{t \in \operatorname{supp} \lambda} \lambda_{t} h_{t}^{*}\left(v_{t}^{*}\right)-\delta_{C}^{*}\left(x^{*}-u^{*}-\sum_{t \in \operatorname{supp} \lambda} \lambda_{t} v_{t}^{*}\right)\right\} \geq \alpha .
\end{aligned}
$$

Therefore, it comes that

$$
\begin{aligned}
& \inf _{x^{*} \in X^{*}} \sup _{\substack{\lambda \in R_{+}^{(T)}, u^{*}, v_{t}^{*} \in X^{*}, t \in \operatorname{supp} \lambda}}\left\{g^{*}\left(x^{*}\right)-f^{*}\left(u^{*}\right)\right. \\
& \left.-\sum_{t \in \operatorname{supp} \lambda} \lambda_{t} h_{t}^{*}\left(v_{t}^{*}\right)-\delta_{C}^{*}\left(x^{*}-u^{*}-\sum_{t \in \operatorname{supp} \lambda} \lambda_{t} v_{t}^{*}\right)\right\} \geq \alpha .
\end{aligned}
$$

Then,

$$
\operatorname{val}(D) \geq \alpha
$$

By Theorem 3.1, we obtain that

$$
\operatorname{val}(P) \geq \alpha,
$$

and the proof is complete.

Finally, in this section, we consider a particular case of the DC problem $(P)$ with $g(x)=0$ when $(P)$ reduces to the following convex programming involving infinite constraints:

$$
\left(P_{0}\right)\left\{\begin{array}{l}
\inf f(x) \\
\text { s.t. } h_{t}(x) \leq 0, t \in T, \text { and } x \in C .
\end{array}\right.
$$


In this case, the dual problem of $\left(P_{0}\right)$ becomes:

$$
\left(D_{0}\right) \sup _{\substack{\lambda \in R_{+}^{(T)}, u^{*}, v_{t}^{*} \in X^{*}, t \in \operatorname{supp} \lambda}}\left\{-f^{*}\left(u^{*}\right)-\sum_{t \in \operatorname{supp} \lambda} \lambda_{t} h_{t}^{*}\left(v_{t}^{*}\right)-\delta_{C}^{*}\left(-u^{*}-\sum_{t \in \operatorname{supp} \lambda} \lambda_{t} v_{t}^{*}\right)\right\} .
$$

Since $g=0$, it follows that dom $g^{*}=\{0\}$. Hence, Theorems 3.4 (resp. 3.5, 3.6) follows from Theorems 3.1 (resp. 3.2, 3.3) directly.

Theorem 3.4. The weak duality between $\left(P_{0}\right)$ and $\left(D_{0}\right)$ is fulfilled, namely, $\operatorname{val}\left(P_{0}\right) \geq \operatorname{val}\left(D_{0}\right)$.

Theorem 3.5. If the condition $(C Q C)$ is fulfilled, then the strong duality between $\left(P_{0}\right)$ and $\left(D_{0}\right)$ holds, namely, val $\left(P_{0}\right)=\operatorname{val}\left(D_{0}\right)$ and the dual problem $\left(D_{0}\right)$ has an optimal solution.

Theorem 3.6. If the condition $(C Q C)$ is satisfied, then, for any $\alpha \in R$, the following statements are equivalent:

(i) $x \in C, h_{t}(x) \leq 0, t \in T \Longrightarrow f(x) \geq \alpha$.

(ii) $(0,-\alpha) \subseteq$ epi $f^{*}+$ cone $\left(\bigcup_{t \in T}\right.$ epi $\left.h_{t}^{*}\right)+$ epi $\delta_{C}^{*}$.

(iii) There exist $\lambda \in R_{+}^{(T)}, u^{*}, v_{t}^{*} \in X^{*}$ and $t \in \operatorname{supp} \lambda$ such that

$$
-f^{*}\left(u^{*}\right)-\sum_{t \in \operatorname{supp} \lambda} \lambda_{t} h_{t}^{*}\left(v_{t}^{*}\right)-\delta_{C}^{*}\left(-u^{*}-\sum_{t \in \operatorname{supp} \lambda} \lambda_{t} v_{t}^{*}\right) \geq \alpha .
$$

4. Applications

Throughout this section, let $X$ and $Y$ be real locally convex Hausdorff topological vector spaces, $C \subseteq X$ be a nonempty convex set. Let $S \subseteq Y$ be a nonempty closed convex cone which defined the partial order of $Y$, namely: $y_{1} \leq_{S} y_{2} \Longleftrightarrow y_{2}-y_{1} \in S$, for any $y_{1}, y_{2} \in Y$. We attach an element $+\infty \notin Y$ which is a greatest element with respect to " $\leq_{S}$ " and let $Y^{\bullet}=Y \cup\{+\infty\}$. The following operations are defined on $Y^{\bullet}: y+(+\infty)=(+\infty)+y=+\infty$ and $t(+\infty)=+\infty$, for any $y \in Y$ and $t \geq 0$. Let $f, g: X \rightarrow \bar{R}$ be two proper convex, and lower semicontinuous functions, and $h: X \rightarrow Y^{\bullet}$ be a proper $S$-convex function. Moreover, we assume that $h$ is star $S$-lower semicontinuous function, that is, the function $(\lambda h)$ is a lower semicontinuous function for any $\lambda \in K^{*}$. Consider the following conic programming problem:

$$
\left(P_{1}\right)\left\{\begin{array}{l}
\inf f(x)-g(x) \\
\text { s.t. } h(x) \in-S, \text { and } x \in C .
\end{array}\right.
$$

This problem has been studied extensively under various degrees of restrictions imposed on $f, g$ and $h$, or on the underlying space, see $[15,16,23,24]$ and the 
references therein. As in [7, 25], for each $\lambda \in K^{*}$, the function $(\lambda h): X \rightarrow \bar{R}$ is defined by

$$
(\lambda h)(x)=\left\{\begin{array}{l}
\langle\lambda, h(x)\rangle, \text { if } x \in \operatorname{dom} h \\
+\infty, \text { otherwise. }
\end{array}\right.
$$

It is easy to see that $h$ is $S$-convex if and only if $(\lambda h)$ is a convex function for each $\lambda \in K^{*}$. Moreover, the problem $(P)_{1}$ can be viewed as an example of $(P)$ by setting

$$
T=S^{*}, h_{\lambda}=\lambda h \text { for any } \lambda \in T=S^{*} .
$$

As before, we use $\mathcal{A}$ to denote the solution set:

$$
\mathcal{A}:=\left\{x \in C:(\lambda h)(x) \leq 0, \forall \lambda \in S^{*}\right\}=\{x \in C: h(x) \in-S\} .
$$

Moreover, since $\bigcup_{\lambda \in S^{*}}$ epi $(\lambda h)^{*}$ is a convex cone (see [37]),

$$
\operatorname{cone}\left(\bigcup_{\lambda \in S^{*}} \operatorname{epi}(\lambda h)^{*}\right)=\bigcup_{\lambda \in S^{*}} \operatorname{epi}(\lambda h)^{*}
$$

and the closedness qualification conditions $(C Q C)$ introduced in Section 3 becomes

$$
(\mathrm{CQC})_{1} \text { epi } f^{*}+\bigcup_{\lambda \in S^{*}} \text { epi }(\lambda h)^{*}+\text { epi } \delta_{C}^{*} \text { is } \text { weak }^{*} \text { closed in } X^{*} \times R .
$$

Note that the regularity condition $(C Q C)_{1}$ was renamed as $(C C C Q)$ or $C_{1}(f ; A)$ or $(C C)$ in other papers, see $[9,15,16]$.

Now, we establish necessary and sufficient conditions for this closedness qualification condition $(C Q C)_{1}$. This condition will be crucial in the sequel and it also deserves some attention for its independent interest.

Lemma 4.1. (See [16]). The following statements are equivalent:

(i) Condition $(C Q C)_{1}$ holds.

(ii) For any $x^{*} \in X^{*}$,

$$
\left(f+\delta_{\mathcal{A}}\right)^{*}\left(x^{*}\right)=\min _{\lambda \in S^{*}, u^{*}, v^{*} \in X^{*}}\left\{f^{*}\left(u^{*}\right)+(\lambda h)^{*}\left(v^{*}\right)+\delta_{C}^{*}\left(x^{*}-u^{*}-v^{*}\right)\right\} .
$$

(iii) For any $\bar{x} \in \mathcal{A} \cap \operatorname{dom} f$ and $\varepsilon \geq 0$, we have the equality

$$
\partial_{\varepsilon}\left(f+\delta_{\mathcal{A}}\right)(\bar{x})=\bigcup_{\lambda \in S^{*}} \bigcup_{\substack{\varepsilon_{1}, \varepsilon_{2}, \varepsilon_{3}, \geq 0, \varepsilon_{1}+\varepsilon_{2}+\varepsilon_{3}=\varepsilon+(\lambda h)(\bar{x})}}\left\{\partial_{\varepsilon_{1}} f(\bar{x})+\partial_{\varepsilon_{2}}(\lambda h)(\bar{x})+N_{\varepsilon_{3}}(C, \bar{x})\right\} .
$$


By the Similar methods of Section 3, we can conduct construct the dual problem of $\left(P_{1}\right)$ as follows:

$$
\left(D_{1}\right) \inf _{x^{*} \in X^{*}} \sup _{\lambda \in S^{*}, u^{*}, v^{*} \in X^{*}}\left\{g^{*}\left(x^{*}\right)-f^{*}\left(u^{*}\right)-(\lambda h)^{*}\left(v^{*}\right)-\delta_{C}^{*}\left(x^{*}-u^{*}-v^{*}\right)\right\} .
$$

Obviously,

$$
\operatorname{val}\left(D_{1}\right)=\inf _{x^{*} \in X^{*}} \operatorname{val}\left(D_{1}^{x^{*}}\right),
$$

where $\left(D_{1}^{x^{*}}\right)$ is defined as

$$
\left(D_{1}^{x^{*}}\right) \sup _{\lambda \in S^{*}, u^{*}, v^{*} \in X^{*}}\left\{g^{*}\left(x^{*}\right)-f^{*}\left(u^{*}\right)-(\lambda h)^{*}\left(v^{*}\right)-\delta_{C}^{*}\left(x^{*}-u^{*}-v^{*}\right)\right\} .
$$

Definition 4.1. We say that

(i) the weak duality between $\left(P_{1}\right)$ and $\left(D_{1}\right)$ holds, if $\operatorname{val}\left(P_{1}\right) \geq \operatorname{val}\left(D_{1}\right)$.

(ii) the strong duality between $\left(P_{1}\right)$ and $\left(D_{1}\right)$ holds, if $\operatorname{val}\left(P_{1}\right)=\operatorname{val}\left(D_{1}\right)$, and for any $x^{*} \in X^{*}$ satisfying $\operatorname{val}\left(D_{1}^{x^{*}}\right)=\operatorname{val}\left(D_{1}\right)$, the dual problem $\left(D_{1}^{x^{*}}\right)$ has an optimal solution.

Remark 4.1. It is easy to see that the strong duality between $\left(P_{1}\right)$ and $\left(D_{1}\right)$ holds if and only if

$$
\operatorname{val}\left(P_{1}\right)=\inf _{x^{*} \in X^{*}} \max _{\lambda \in S^{*}, u^{*}, v^{*} \in X^{*}}\left\{g^{*}\left(x^{*}\right)-f^{*}\left(u^{*}\right)-(\lambda h)^{*}\left(v^{*}\right)-\delta_{C}^{*}\left(x^{*}-u^{*}-v^{*}\right)\right\} .
$$

Theorem 4.1 (resp. 4.2, 4.3) can be derived from Theorems 3.1 (resp. 3.2, 3.3) directly. These results were recently established in $[15,16]$.

Theorem 4.1. The weak duality between $\left(P_{1}\right)$ and $\left(D_{1}\right)$ is fulfilled, namely, $\operatorname{val}\left(P_{1}\right) \geq \operatorname{val}\left(D_{1}\right)$.

Theorem 4.2. If the condition $(C Q C)_{1}$ is fulfilled, then the strong duality between $\left(P_{1}\right)$ and $\left(D_{1}\right)$ holds, namely, $\operatorname{val}\left(P_{1}\right)=\operatorname{val}\left(D_{1}\right)$, and for any $x^{*} \in X^{*}$ satisfying $\operatorname{val}\left(D_{1}^{x^{*}}\right)=\operatorname{val}\left(D_{1}\right)$, the dual problem $\left(D_{1}^{x^{*}}\right)$ has an optimal solution.

Theorem 4.3. If the condition $(C Q C)_{1}$ is satisfied, then, for any $\alpha \in R$, the following statements are equivalent:

(i) $x \in C, h(x) \in-S \Longrightarrow f(x)-g(x) \geq \alpha$.

(ii) $(0,-\alpha)+$ epi $g^{*} \subseteq$ epi $f^{*}+\bigcup_{\lambda \in S^{*}}$ epi $(\lambda h)^{*}+$ epi $\delta_{C}^{*}$.

(iii) For any $x^{*} \in X^{*}$, there exist $\lambda \in S^{*}, u^{*}, v^{*} \in X^{*}$ such that

$$
g^{*}\left(x^{*}\right)-f^{*}\left(u^{*}\right)-(\lambda h)^{*}\left(v^{*}\right)-\delta_{C}^{*}\left(x^{*}-u^{*}-v^{*}\right) \geq \alpha .
$$


Finally, in this section, we consider a particular case of the problem $\left(P_{1}\right)$ with $g(x)=0$ when $\left(P_{1}\right)$ reduces to the following convex programming:

$$
\left(P_{2}\right)\left\{\begin{array}{l}
\inf f(x) \\
\text { s.t. } h(x) \in-S, \text { and } x \in C .
\end{array}\right.
$$

In this case, the dual problem of $\left(P_{0}\right)$ becomes:

$$
\left(D_{2}\right) \sup _{\lambda \in S^{*}, u^{*}, v^{*} \in X^{*}}\left\{-f^{*}\left(u^{*}\right)-(\lambda h)^{*}\left(v^{*}\right)-\delta_{C}^{*}\left(-u^{*}-v^{*}\right)\right\} .
$$

Similarly, we can easily obtain the following theorems. These results were recently established in $[25,26]$.

Theorem 4.4. The weak duality between $\left(P_{2}\right)$ and $\left(D_{2}\right)$ is fulfilled, namely val $\left(P_{2}\right)$ $\geq \operatorname{val}\left(D_{2}\right)$.

Theorem 4.5. If the condition $(C Q C)_{1}$ is fulfilled, then the strong duality between $\left(P_{2}\right)$ and $\left(D_{2}\right)$ holds, namely val $\left(P_{2}\right)=\operatorname{val}\left(D_{2}\right)$ and the dual problem $\left(D_{2}\right)$ has an optimal solution.

Theorem 4.6. If the condition $(C Q C)_{1}$ is satisfied, then, for any $\alpha \in R$, the following statements are equivalent:

(i) $x \in C, h(x) \in-S \Longrightarrow f(x) \geq \alpha$.

(ii) $(0,-\alpha) \subseteq$ epi $f^{*}+\bigcup_{\lambda \in S^{*}}$ epi $(\lambda h)^{*}+$ epi $\delta_{C}^{*}$.

(iii) There exist $\lambda \in S^{*}, u^{*}, v^{*} \in X^{*}$ such that

$$
-f^{*}\left(u^{*}\right)-(\lambda h)^{*}\left(v^{*}\right)-\delta_{C}^{*}\left(-u^{*}-v^{*}\right) \geq \alpha .
$$

\section{CONCLUSION}

In this paper, we first introduce some closedness qualification conditions for a DC infinite programming problem with inequality constraints. We also recall some characterizations of these closedness qualification conditions. Then, by using these constraint qualifications, we investigate the duality results and Farkas-type results for this DC infinite programming problem. Our results generalize and rediscover some results in cone-convex optimization problems.

\section{ACKNOWLEDGMENTS}

The authors would like to thank the anonymous reviewers for valuable comments and suggestions, which helped to improve the paper. 


\section{REFERENCES}

1. E. Polak, Optimization. Algorithms and Consistent Approximations, Applied Mathematical Sciences, Springer, New York, 1997.

2. M. Goberna and M. López, Linear Semi-Infinite Optimization, Wiley, Chichester, 1998.

3. J. Bonnans and A. Shapiro, Perturbation Analysis of Optimization Problems, Springer Series in Operations Research, Springer, New York, 2000.

4. O. Stein and G. Still, On optimality conditions for generalized semi-infinite programming problems, J. Optim. Theory Appl., 104 (2000), 443-458.

5. G. Stein, Solving generalized semi-infinite programs by reduction to simpler problems, Optimization, 53 (2004), 19-38.

6. R. I. Boț and G. Wanka, Farkas-type results with conjugate functions, SIAM J. Optim., 15 (2005), 540-554.

7. R. I. Boț, Conjugate Duality in Convex Optimization, Springer-Verlag, Berlin, 2010.

8. N. Dinh, M. A. Goberna, M. A. López and T. Q. Song, New Farkas-type constraint qualifications in convex infinite programming, ESAIM Control Optim. Calc. Var., 13 (2007), 580-597.

9. D. H. Fang, C. Li and K. F. Ng, Constraint qualifications for extended Farkas's lemmas and Lagrangian dualities in convex infinite programming, SIAM J. Optim., 20 (2009), 1311-1332.

10. D. H. Fang, C. Li and K. F. Ng, Constraint qualifications for optimality conditions and total Lagrange dualities in convex infinite programming, Nonlinear Anal., 73 (2010), 1143-1159.

11. M. A. Goberna, V. Jeyakumar and M. A. López, Necessary and sufficient conditions for solvability of systems of infinite convex inequalities, Nonlinear Anal., 68 (2008), 1184-1194.

12. J. E. Martinez-Legaz and M. Volle, Duality in DC programming: The case of several DC constraints, J. Math. Anal. Appl., 237 (1999), 657-671.

13. R. I. Boț, I. B. Hodrea and G. Wanka, Some new Farkas-type results for inequality system with DC functions, J. Glob. Optim., 39 (2007), 595-608.

14. R. I. Boţ, I. B. Hodrea and G. Wanka, Farkas-type results for fractional programming problems, Nonlinear Anal., 67 (2007), 1690-1703.

15. N. Dinh, G. Vallet and T. T. A. Nghia, Farkas-type results and duality for DC programs with convex constraints, J. Convex Anal., 15 (2008), 235-262.

16. N. Dinh, T. T. A. Nghia and G. Vallet, A closedness condition and its applications to DC programs with convex constraints, Optimization, 59 (2010), 541-560.

17. D. H. Fang, C. Li and X. Q. Yang, Stable and total Fenchel duality for DC optimization problems in locally convex spaces, SIAM J. Optim., 21 (2011), 730-760. 
18. X. K. Sun and S. J. Li, Duality and Farkas-type results for extended Ky Fan inequalities with DC functions, Optim. Lett., 7 (2013), 499-510.

19. D. H. Fang, C. Li and X. Q. Yang, Asymptotic closure condition and Fenchel duality for DC optimization problems in locally convex spaces, Nonlinear Anal., 75 (2012), 3672-3681.

20. D. H. Fang, G. M. Lee, C. Li and J. C. Yao, Extended Farkas's lemmas and strong Lagrange dualities for DC infinite programming, J. Nonlinear Convex Anal., to appear.

21. V. F. Demyanov and A. M. Rubinov, Constructive Nonsmooth Analysis, Peter Lang, Frankfurt, 1995.

22. R. T. Rockafellar and R. J. Wets, Variational Analysis, Springer-Verlag, Berlin, 1998.

23. N. Dinh, B. S. Mordukhovich and T. T. A. Nghia, Subdifferentials of value functions and optimality conditions for DC and bilevel infinite and semi-infinite programs, Math. Program., 123 (2010), 101-138.

24. N. Dinh, B. S. Mordukhovich and T. T. A. Nghia, Qualification and optimality conditions for convex and DC programs with infinite constraints, Acta Math. Vietnamica, 34 (2009), 123-153.

25. R. I. Boţ, S. M. Grad and G. Wanka, On strong and total Lagrange duality for convex optimization problems, J. Math. Anal. Appl., 337 (2008), 1315-1325.

26. R. I. Boţ, S. M. Grad and G. Wanka, New regularity conditions for strong and total Fenchel-Lagrange duality in infinite dimensional spaces, Nonlinear Anal., 69 (2008), 323-336.

27. V. Jeyakumar, N. Dinh and G. M. Lee, A New Closed Cone Constraint Qualification for Convex Optimization, Applied Mathematics Report AMR 04/8, University of New South Wales, 2004.

28. V. Jeyakumar, W. Song, N. Dinh and G. M. Lee, Stable Strong Duality in Convex Optimization, Applied Mathematics Report AMR 05/22, University of New South Wales, 2005.

29. R. S. Burachik and V. Jeyakumar, A new geometric condition for Fenchel's duality in infinite dimensional spaces, Math. Program., 104 (2005), 229-233.

30. R. I. Boț and G. Wanka, A weaker regularity condition for subdifferential calculus and Fenchel duality in infinite dimensional spaces, Nonlinear Anal., 64 (2006), 2787-2804.

31. V. Jeyakumar, Constraint qualifications characterizing Lagrangian duality in convex optimization, J. Optim. Theory Appl., 136 (2008), 31-41.

32. R. I. Boț, S. M. Grad and G. Wanka, A new constraint qualification for the formula of the subdifferential of composed convex functions in infinite dimensional spaces, Math. Nachr., 281 (2008), 1-20.

33. V. Jeyakumar and G. M. Lee, Complete characterization of stable Farkas lemma and cone-convex programming duality, Math. Program., 114 (2008), 335-347. 
34. V. Jeyakumar, G. M. Lee and N. Dinh, New sequential Lagrange multiplier conditions characterizing optimality without constraint qualifications for convex programs, SIAM J. Optim., 14 (2003), 534-547.

35. J. F. Toland, Duality in nonconvex optimization, J. Math. Anal. Appl., 66 (1978), 399415.

36. C. Zalinescu, Convex Analysis in General Vector Spaces, World Scientific, River Edge, NJ, 2002.

37. V. Jeyakumar, A. Rubinov, B. M. Glover and Y. Ishizuka, Inequality systems and global optimization, J. Math. Anal. Appl., 202 (1996), 900-919.

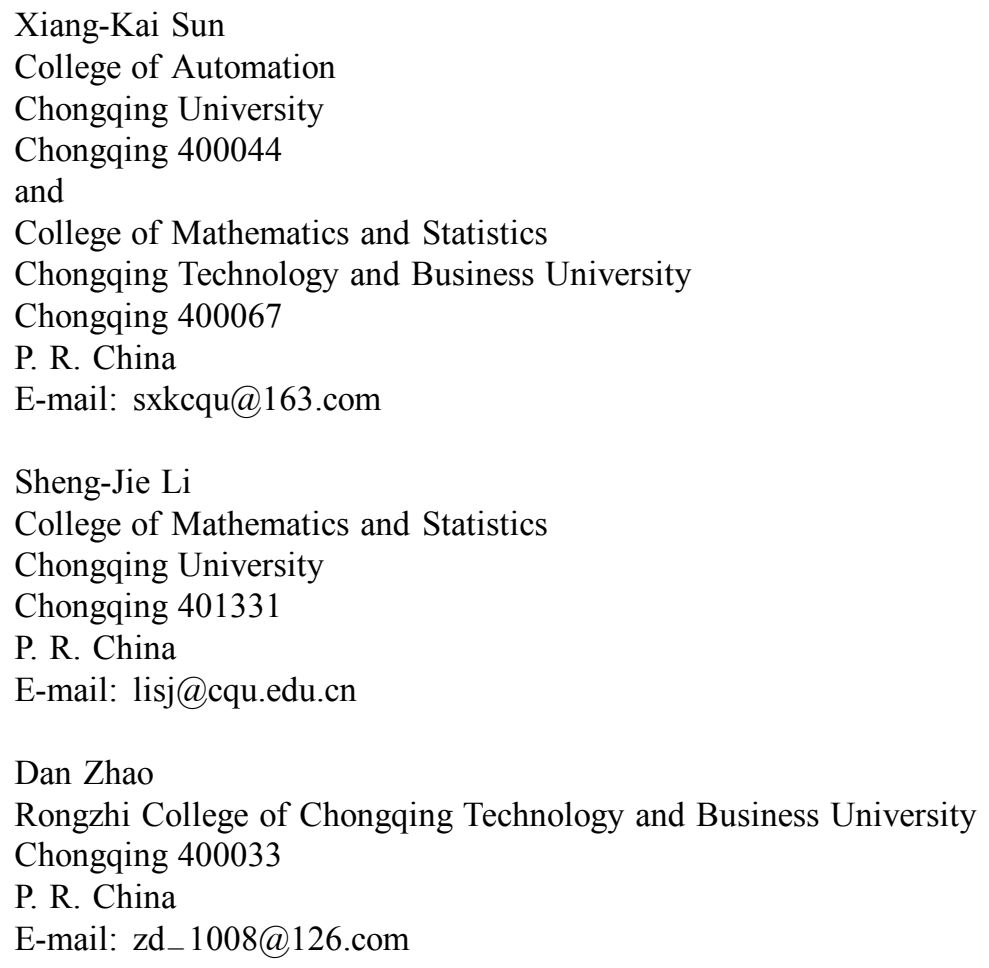

